\title{
Subclinical Hypothyroidism in Children and Adolescents: Is It Clinically Relevant?
}

\author{
Aneta Gawlik, ${ }^{1}$ Kamila Such, ${ }^{2}$ Aleksandra Dejner, ${ }^{2}$ Agnieszka Zachurzok, \\ Aleksandra Antosz, ${ }^{3}$ and Ewa Malecka-Tendera ${ }^{1}$ \\ ${ }^{1}$ Department of Pediatrics, Pediatric Endocrinology and Diabetes, School of Medicine in Katowice, Medical University of Silesia, \\ 40752 Katowice, Poland \\ ${ }^{2}$ Medical Students' Scientific Association, 40752 Katowice, Poland \\ ${ }^{3}$ Department of Pediatrics, Pediatric Endocrinology and Diabetes, Upper-Silesian Pediatric Health Center, 40752 Katowice, Poland
}

Correspondence should be addressed to Aneta Gawlik; agawlik@mp.pl

Received 30 November 2014; Revised 4 February 2015; Accepted 27 February 2015

Academic Editor: Andreas Tomaschitz

Copyright (C) 2015 Aneta Gawlik et al. This is an open access article distributed under the Creative Commons Attribution License, which permits unrestricted use, distribution, and reproduction in any medium, provided the original work is properly cited.

\begin{abstract}
Although subclinical hypothyroidism (SH) is a common clinical problem, its diagnosis tends to be incidental. According to the definition, it should be asymptomatic, only detectable by screening. The presence or coincidence of any symptoms leads to L-thyroxine treatment. The clinical presentation, especially in younger patients with subclinical hypothyroidism, is still under dispute. Accordingly, the aim of this paper was to review the literature from the past seven years. The literature search identified 1,594 potentially relevant articles, of which 24 met the inclusion criteria. Few studies focus on the symptomatology of subclinical hypothyroidism, and most of them analyzed a small number of subjects. A significant correlation was found by some authors between subclinical hypothyroidism and a higher risk of hypertension, dyslipidemia, and migraine. No evidence of the impact of subclinical hypothyroidism on weight, growth velocity, and puberty was revealed. As the quality of most studies is poor and no definite conclusions can be drawn, randomized, large-scale studies in children and adolescents are warranted to determine the best care for patients with $\mathrm{SH}$.
\end{abstract}

\section{Introduction}

From the biochemical point of view, subclinical hypothyroidism (SH) is characterized by mildly elevated serum TSH concentrations, with normal concentrations of serum free and total triiodothyronine (T3) and thyroxine (T4), without the typical symptoms of thyroid disease. $\mathrm{SH}$ prevalence in adults ranges from 4 to $10 \%$ [1]. In the pediatric population, the prevalence of this thyroid disorder is estimated to be less than $10 \%$ [2]. According to Biondi and Cooper, children with $\mathrm{SH}$ can present some minimal or nonspecific signs and symptoms [1]. There is a paucity of long-term prospective research studying the natural history of subclinical hypothyroidism and its consequences in childhood [3,4]. A preliminary $\mathrm{SH}$ diagnosis is confirmed by laboratory test when TSH concentration is above the statistically defined upper limit of the reference range [5].
In the adult population with subclinical thyroid disease, $\mathrm{SH}$ is associated with a risk of progression to overt thyroid disease, lipid disorders, increased risk of atherosclerosis, and mortality due to cardiovascular diseases [6]. The public data regarding the clinical manifestation of $\mathrm{SH}$ in children and adolescents are inconsistent as most papers indicate $\mathrm{SH}$ to be asymptomatic [7].

Accordingly, the aim of this paper was to analyze studies reporting signs and symptoms presented by children and adolescents diagnosed with subclinical hypothyroidism.

\section{Methodology}

2.1. Literature Search. In order to identify studies evaluating the clinical manifestations and symptoms of subclinical hypothyroidism in children, a systematic PubMed literature search was conducted. The search terms used in 
the medical subject headings (MeSH) included (subclinical [All Fields] AND ("hypothyroidism" [MeSH Terms] OR "hypothyroidism" [All Fields])) as well as (subclinical [All Fields] AND ("hypothyroidism" [MeSH Terms] OR "hypothyroidism" [All Fields]) AND ("infant" [MeSH Terms] OR “child” [MeSH Terms] OR “adolescent” [MeSH Terms])) and SH hypothyroidism [All Fields] AND "humans" [MeSH Terms] AND ("infant" [MeSH Terms] OR "child" [MeSH Terms] OR "adolescent" [MeSH Terms]) and hyperthyrotropinemia [All Fields] AND "humans" [MeSH Terms] AND ("infant" [MeSH Terms] OR "child" [MeSH Terms] OR "adolescent" [MeSH Terms]). These terms were combined in various ways to generate a wider search.

In addition, the references of selected articles were checked with a view to identify papers not detected by our search strategy. Only full-length publications that met the following criteria were included: (1) long-term prospective or retrospective studies regarding the clinical signs of $\mathrm{SH}$ in the pediatric cohort; (2) only studies in English; (3) studies published between January 2008 and December 2014. Exclusion criteria were the following: (1) studies including patients with chronic systematic diseases, genetic syndromes, or autoimmune disorders or under concomitant therapy with lithium salts, antiepileptic agents, glucocorticoids, or iodinated drugs; (2) studies regarding the treatment and effects of L-thyroxine replacement therapy. At least two authors independently selected articles for inclusion and exclusion criteria (Figure 1).

\section{Results}

After reviewing 1,594 titles, abstracts, and full-length texts, 24 articles meeting the inclusion criteria were selected for the final analysis [8-31]. There were sixteen cross-sectional studies $[9,10,12-15,17-19,21-27,29,30]$, twelve studies that met the criteria of case-control trials $[9,11,14,18-20,23-$ $25,27,29,30]$, ten longitudinal trials $[9-15,17,21,23,25]$, and four retrospective studies [8, 16, 28, 31]. Overall, data from a total of 139756 children were reported. Most studies included small numbers of children; only one retrospective study described a large population [8]. The subjects' ages ranged from 2 weeks to 18 years, whilst the follow-up after $\mathrm{SH}$ diagnosis was from 2 weeks to 108 months. The cut-off limits for TSH concentrations were between 3.0 and $6.7 \mathrm{mIU} / \mathrm{L}$ and $\leq 10 \mathrm{mIU} / \mathrm{L}$. Studies analyzing the clinical manifestations of $\mathrm{SH}$ are demonstrated in Table 1 .

3.1. SH and Weight/BMI. In their cross-sectional controlled long-term study of 36 children with SH and controls, Cerbone et al. [9] assessed the relationship between weight/BMI and persistent untreated $\mathrm{SH}$ during follow-up. The authors concluded that only three (8\%) SH children were obese. No significant alterations in weight or BMI were noticed during the period from diagnosis to study enrollment $(3.3 \pm 0.3$ yrs). Similar observations concerning stable BMI values were presented by Wasniewska et al. [10].

In a case-control study, Kuiper and van der Gaag [11] demonstrated the impact of a diet rich in iron, iodine,

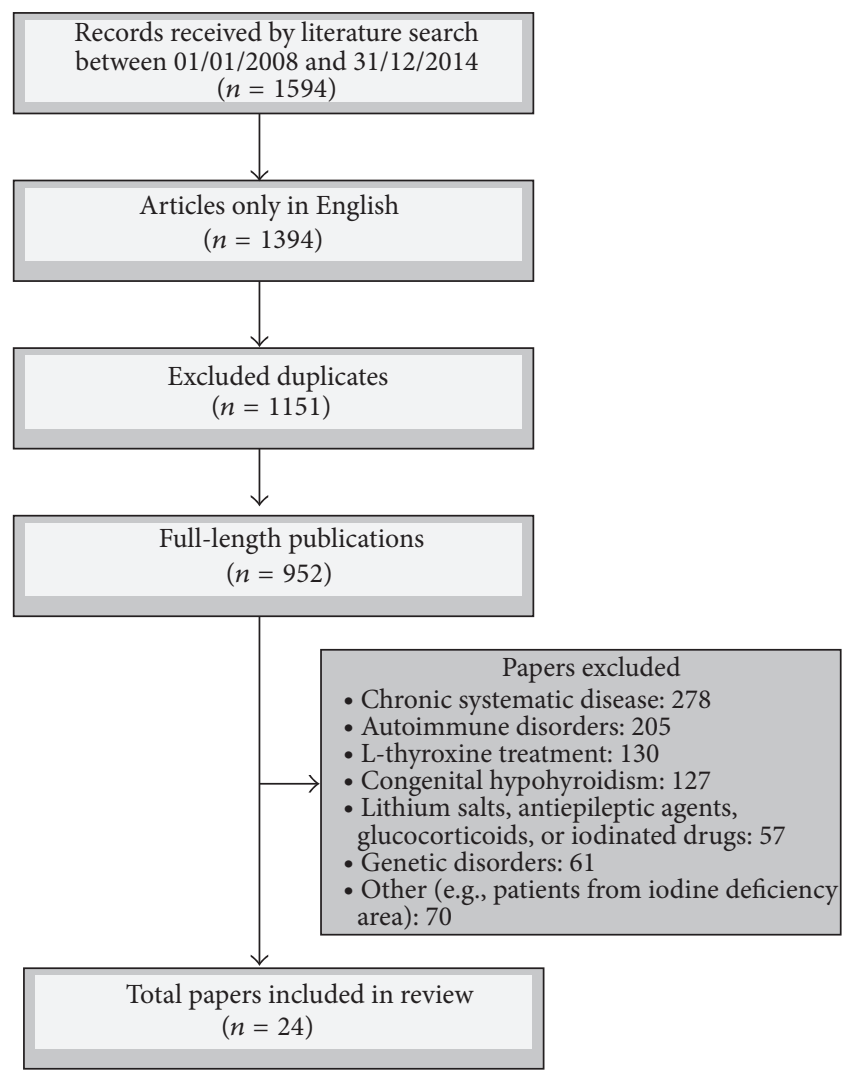

FIgURE 1: Study design and inclusion/exclusion criteria.

and vitamin A. The authors hypothesized that the main problem regarding $\mathrm{SH}$ in children was an immunological dysfunction or deficiency of micronutrients, which cause disturbed thyroid hormone production. The study encompassed 54 children with $\mathrm{SH}$, aged 1-14 yrs, divided into 2 groups: diet and control. The 3-month diet consisted of green vegetables, beef, full fat milk, and butter. TSH, FT4, and BMI were evaluated during the follow-up. The results showed a significant drop in TSH in the diet group. The limitations of the study were a small number of patients and a nonrandomized study design.

Some studies have confirmed the relationship between the levels of TSH and FT3 and change of body weight. The level of FT4 remained stable during follow-up. Leptin was hypothesized to link the weight status with TSH. This would suggest that TSH and FT3 concentrations in obese subjects are a consequence rather than the cause of obesity [12-15].

Reinehr et al. [12] analyzed thyroid hormones in female adolescents with obesity and anorexia nervosa before and after weight normalization. The authors determined the serum levels of TSH, FT3, and FT4 in 100 obese girls, 32 normal-weight girls, and 20 girls with anorexia nervosa aged 14-18 yrs. The measurements were carried out at baseline and after 1 year. Additionally, leptin, insulin, and homeostasis model assessment (HOMA) index were analyzed in obese and normal-weight girls in order to determine the degree of insulin resistance. The results revealed that, compared to 


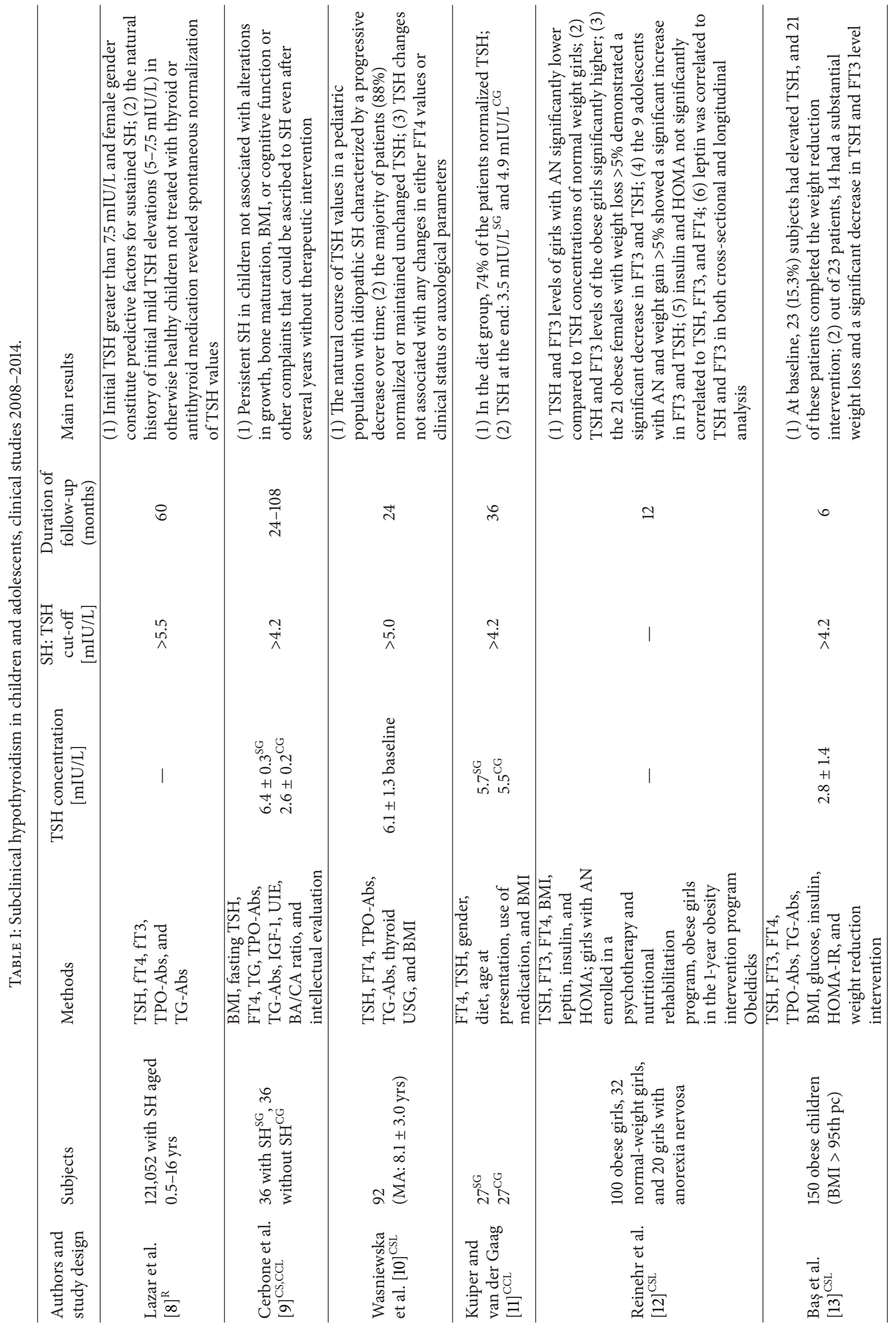




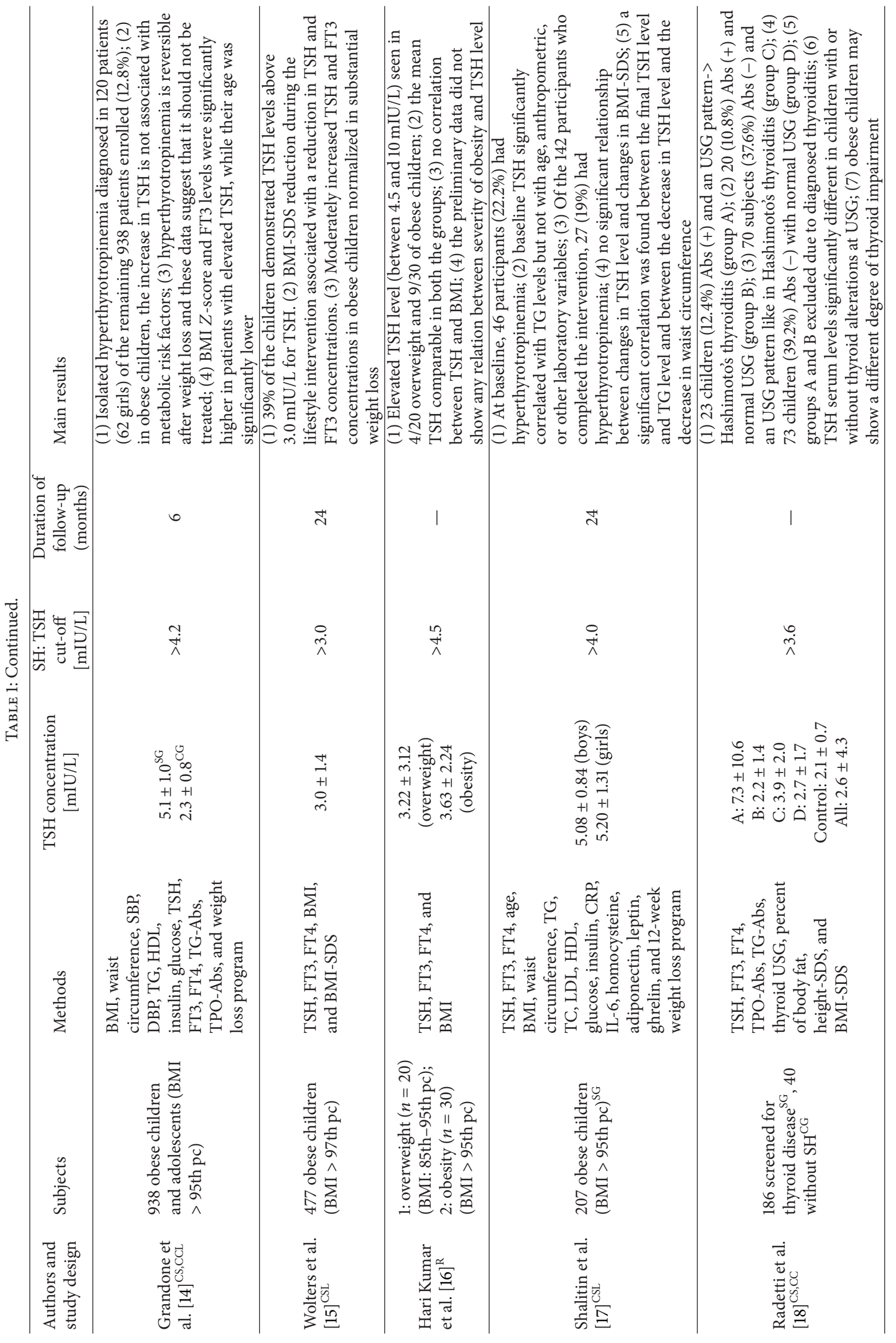




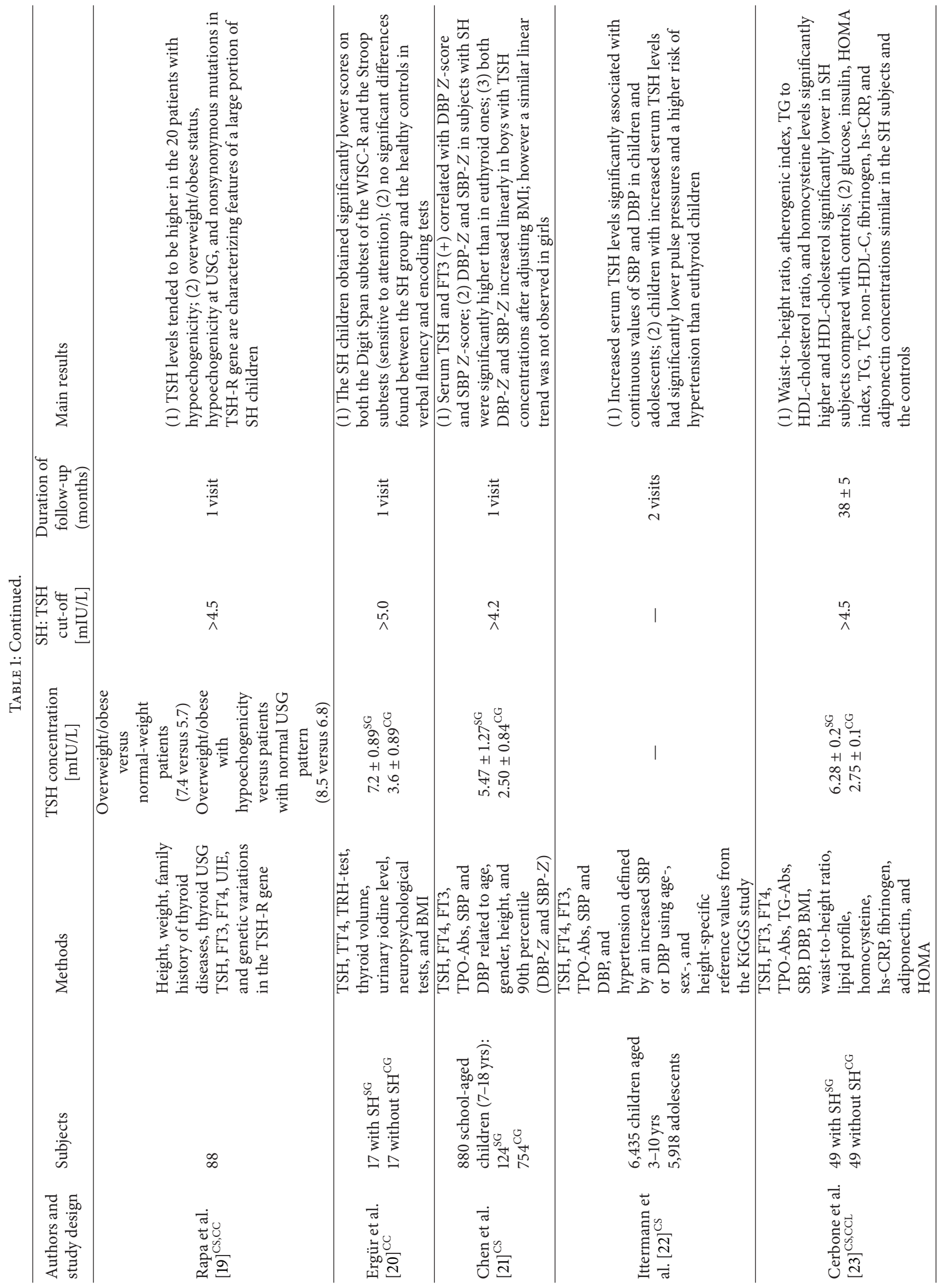




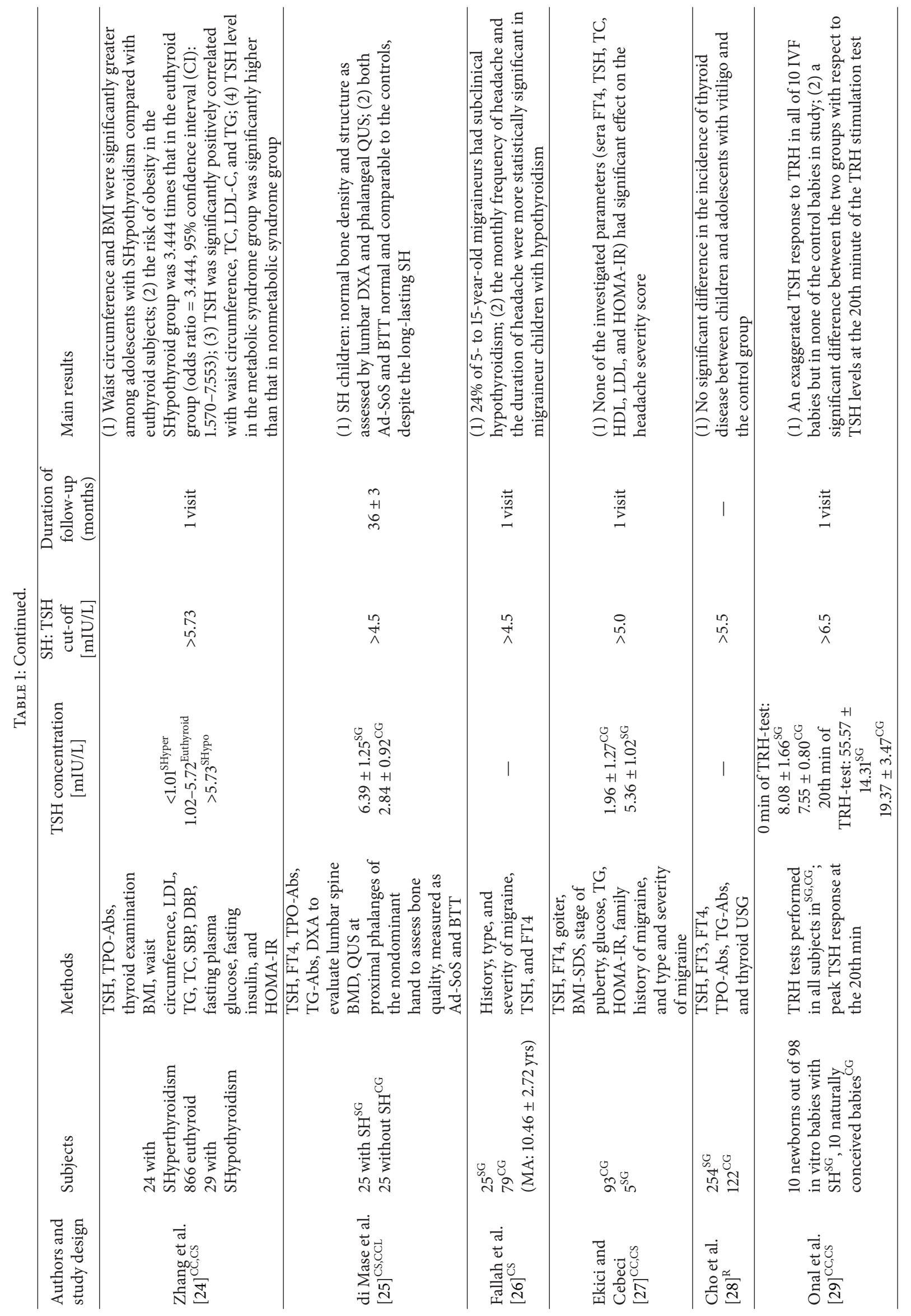




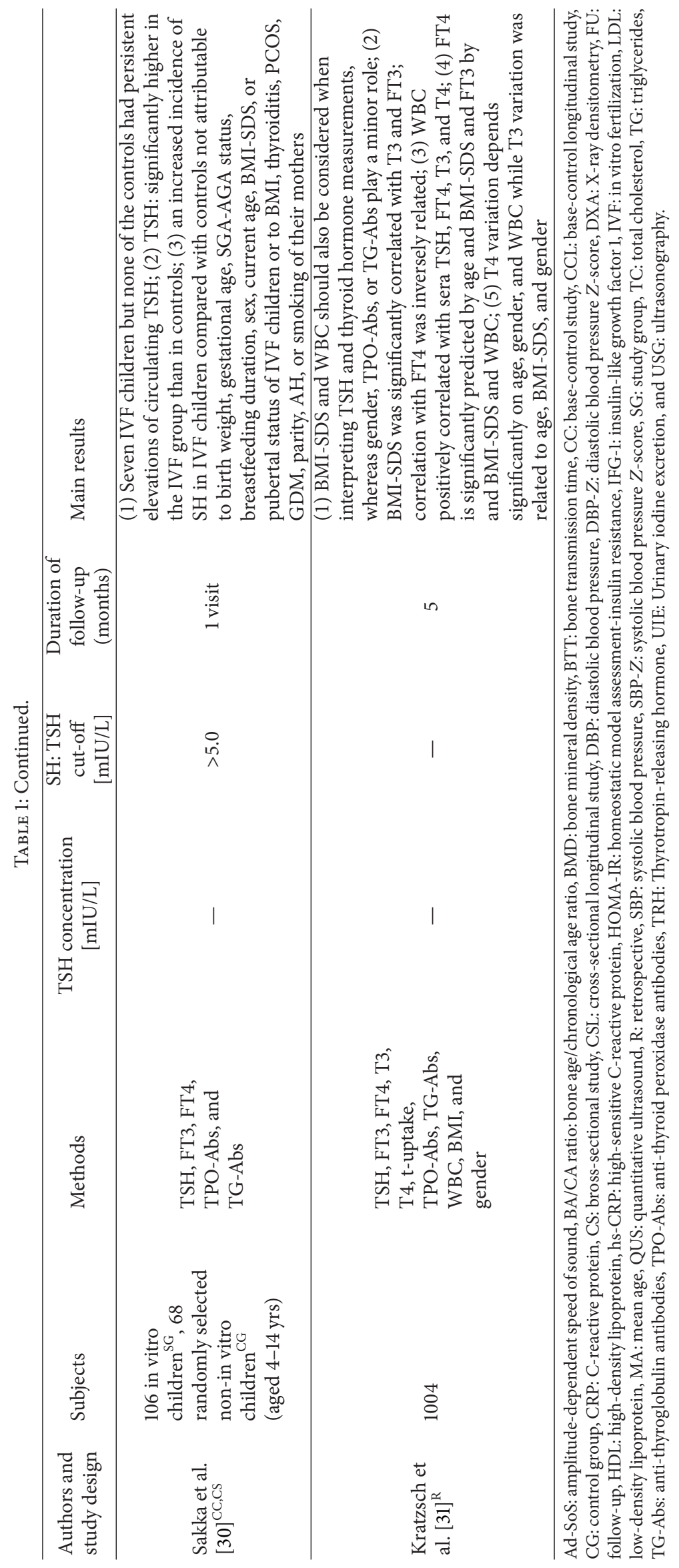


normal-weight girls, the levels of TSH and FT3 were significantly lower in girls with anorexia nervosa and significantly higher in the obese. The obese patients who lost more than $5 \%$ of their weight demonstrated a valid decrease in FT3 and TSH, whilst patients with anorexia nervosa who gained weight showed a notable increase in FT3 and TSH. Unlike leptin, HOMA and insulin were not correlated to TSH, FT3, and FT4.

Similar conclusions were drawn by Baş et al. [13], who observed a positive correlation between weight loss and normalization of serum TSH levels.

The study by Grandone et al. [14] encompassed 938 obese children and adolescents (450 females). The aims of the study were to (1) ascertain the spread of abnormally elevated TSH levels in young obese Italian patients, (2) determine if highly elevated levels of TSH in obese children could constitute cardiovascular and metabolic risk factors, and (3) to check if elevated TSH is reversible after weight loss. Anthropometric, metabolic, and hormonal variables were determined at baseline and, in a subgroup of children with hyperthyrotropinemia, after a six-month weight-loss program. The results suggest that (1) a moderate elevation of TSH concentrations is common in obese children, (2) hyperthyrotropinemia is not connected with metabolic risk factors in obese patients, and (3) moderate elevation of TSH is reversible after weight loss, and, according to the authors, no treatment is needed.

The association between TSH, FT3, FT4, and weight status, as well as their changes during and after a lifestyle intervention in obese children, was also studied by Wolters et al. [15]. They evaluated the weight status/BMI-SDS in 477 obese children who took part in a 1-year lifestyle intervention in a 2-year longitudinal study. At baseline, 39\% of the children had TSH level $>3.0 \mathrm{mIU} / \mathrm{L}$. A reduction in BMISDS (>0.5) during the intervention was associated with a decrease and normalization of TSH and FT3 concentrations. Interestingly, a decrease in TSH and FT3 concentrations by lifestyle intervention was at the same time associated with an increase in BMI-SDS after the intervention time.

Two other studies investigating the correlation between BMI in obese children and hyperthyrotropinemia have produced interesting findings. Hari Kumar et al. [16] compared the TSH level in a cohort of 50 children divided into two groups: overweight and obese. The TSH levels were comparable in both groups and the authors did not find a significant impact of the degree of obesity on the level of serum TSH. Shalitin et al. [17] investigated the potential relationship between the levels of serum TSH and other metabolic and hormonal variables before and after weight loss. They enrolled 207 obese children and adolescents aged 5-18 yrs who underwent biochemical, hormonal, anthropometric, and metabolic examinations before and after weight reduction. Hyperthyrotropinemia was confirmed in $46(22.2 \%)$ participants and correlated positively with higher triglycerides. The decrease in TSH concentration correlated positively with a reduction in waist circumference. No correlation between TSH and leptin levels was confirmed. All the patients had normal FT4 concentrations.
The investigators raised the question of the necessity to treat hyperthyrotropinemia in obese children.

Radetti et al. [18] investigated the pathological structure/morphology of the thyroid gland and its function in obese children. The cross-sectional study included 186 overweight and obese young patients. Ultrasonography, FT3, FT4, $\mathrm{TSH}$, and antithyroid antibodies were performed in all subjects. The control group was composed of 40 healthy children. The cohort was divided into four groups: A: with Hashimoto disease, B: with positive antibodies and normal ultrasound, C: with negative antibodies and ultrasound pattern suggestive of Hashimoto's thyroiditis, and D: with negative antibodies and normal ultrasound. Groups A and C had higher TSH levels than groups $\mathrm{B}$ and $\mathrm{D}$ and controls. The study showed that alterations in thyroid structure and function in obese patients are quite common. Obesity is often associated with a lowgrade inflammatory state, which would explain the abnormal ultrasound of the thyroid gland in patients with large deposits of adipose tissue.

The multicenter study by Rapa et al. [19] was based on 88 children and adolescents with $\mathrm{SH}$ diagnosed by at least two determinations of TSH above the upper limit of the reference range. The prevalence of overweight/obesity in the study group was $28.4 \%$. Overweight and obese patients had significantly higher serum TSH levels than patients with normal weight (7.4 versus $5.7 \mathrm{IU} / \mathrm{mL}$ ); the highest values were found in patients with hypoechogenicity in thyroid ultrasonography $(8.5 \mathrm{IU} / \mathrm{mL})$. The multiple regression analysis showed that increased serum TSH concentrations (multiple $R=0.31$; $P=0.02$ ) were independently caused by both younger age and overweight/obesity status.

3.2. SH and Intellectual Outcomes. In a study that included $30 \mathrm{SH}$ children and 36 controls, Cerbone et al. [9] evaluated verbal, performance, and full-scale intelligence quotient (IQ) scores as well as the children's psychological development. The intellectual outcomes were normal for the children's age and comparable to controls. There was no relationship between mean serum TSH concentrations during the observation or the persistence of SH and IQ scores, IQ subtests, age-appropriate child behavior checklist, and Children's Depression Inventory scores.

Ergür et al. [20] conducted a case-control study including 17 children with SH and 17 healthy children with TSH serum levels $7.2 \pm 0.89 \mathrm{mUI} / \mathrm{L}$ versus $3.6 \pm 0.89 \mathrm{mUI} / \mathrm{L}$, respectively. Analyzing the cognitive functions, such as active/passive attention, maintaining attention, and response inhibition, the authors reported significantly lower scores in the $\mathrm{SH}$ group than in controls, both on the Digit Span subtest of the Wechsler Intelligence Scale for Children-Revised (WISC-R) and the Stroop subtests. No significant differences were found between the $\mathrm{SH}$ group and the healthy controls in verbal fluency and encoding tests.

3.3. SH Blood Pressure and Cardiovascular Risk Factors. In their cross-sectional trial, Chen et al. [21] investigated the correlation between serum TSH levels and blood pressure in Chinese school-aged children without overt thyroid disease. 
Of 880 enrolled children, 127 (14.4\%) were diagnosed with subclinical hypothyroidism. During the 7-month follow-up, both systolic and diastolic blood pressure $Z$-scores were found to be higher in subjects with subclinical hypothyroidism than in euthyroid subjects; in males, the scores increased linearly with increasing TSH.

Ittermann et al. [22] evaluated the relationship between serum TSH concentrations, pulse, and systolic and diastolic blood pressure in 12,353 subjects divided into two groups: children (3-10 yrs) and adolescents (11-17 yrs). Subclinical hypothyroidism was observed in 5\% of the study population and was associated with continuous values of systolic and diastolic blood pressure in children and adolescents. The results suggest that $\mathrm{SH}$ is associated with an increased risk of hypertension. Although the pathophysiological mechanism underlying this finding is not well understood, authors speculate that it may be due to endothelial dysfunction, left ventricular hypertrophy, or thickened arterial walls. No relationship was observed between Tanner stage IV/V in adolescents and TSH concentration. Furthermore, adolescents with $\mathrm{SH}$ had lower pulse rate than euthyroid subjects.

Cerbone et al. [23] in a cross-sectional and controlled study evaluated the clinical and biochemical cardiovascular risk factors in untreated children with mild $\mathrm{SH}$ (TSH serum concentration $4.5-10 \mathrm{mU} / \mathrm{L}$ ). After two years of the follow-up, children with $\mathrm{SH}$ had significantly higher waistto-hip ratio, triglycerides to high-density-lipoprotein ratio, and homocysteine level and lower high-density lipoproteincholesterol. The authors concluded that mild long-lasting SH may be associated with subtle proatherogenic abnormalities although these changes may not represent the early stage of atherogenesis.

Positive correlation between the components of the metabolic syndrome and serum TSH was also found in Chinese adolescents by Zhang et al. [24].

3.4. SH and Linear Growth, Bone Maturation. In their crosssectional controlled long-term trial, Cerbone et al. [9] also analyzed growth in children with persistent untreated $\mathrm{SH}$. The parameters of height and bone age/chronological age were within normal ranges at the beginning of the trial and did not deteriorate over several years of follow-up. Despite the normal mean height, a subgroup of $8 / 36$ (22\%) $\mathrm{SH}$ children had short stature. Six of them were within the target height range, thus suggesting familial short stature; the other two $(5.5 \%)$ had stature below the target height with normal growth velocity, normal IGF1 concentration, GH peak after stimulation test within the reference range, and a delay of 2 years in bone maturation, indicating a possible constitutional delay of growth and puberty. None of the parameters deteriorated over several years of follow-up in $\mathrm{SH}$ children and controls.

di Mase et al. [25] in their cross-sectional prospective trial evaluated the possible effect of untreated $\mathrm{SH}$ on bone health in childhood. Two methods were used: dual-energy Xray densitometry (DXA) and quantitative ultrasound (QUS). The authors recruited 25 subjects with $\mathrm{SH}$ and 25 randomly chosen age- and sex-matched healthy children as controls.
All values of lumbar spine DXA and phalangeal QUS were within the normal range, both in patients and in controls, despite the long-term duration of untreated SH. The statistical analysis showed that neither TSH levels at the beginning of the study nor the duration of $\mathrm{SH}$ had a significant impact on bone mineral density (BMD), amplitude-dependent speed of sound (Ad-SoS), and bone transmission time $Z$-scores (BTT $Z$-scores).

3.5. SH and Migraine. One cross-sectional study conducted by Fallah et al. [26] assessed the monthly prevalence of headache in children with subclinical thyroid disease. The authors concluded that children aged between 5 and 15 yrs diagnosed with $\mathrm{SH}$ suffered more frequently from the migraines which were also lasting longer. The authors suggest checking the thyroid function in migraineur children and adolescents. These findings were not confirmed by Ekici and Cebeci [27] who detected subclinical hypothyroidism only in $5 \%$ of children with headache. They concluded that initial endocrinological evaluation or screening for $\mathrm{SH}$ in children suffering from migraine is unnecessary.

3.6. Natural Course of SH. Lazar et al. [8] presented the natural history of thyroid function tests over five years in a large pediatric cohort. The authors analyzed the survey demographic data, referral diagnoses, and laboratory results (TSH, FT4, and thyroid antibodies) of 121,052 children with $\mathrm{SH}$ aged $0.5-16$ yrs. First, the authors determined the relative proportion of regular and irregular thyroid function results in the cohort. Next, they established the natural history of initial abnormal TSH levels in otherwise healthy children without any thyroid disease. Finally, they defined the number of patients at an increased risk for developing subsequent abnormal TSH levels. Normal initial TSH concentrations were observed in $96.5 \%$ of children; $0.2 \%$ had low TSH concentrations ( $0.35 \mathrm{mIU} / \mathrm{L}), 2.9 \%$ elevated $(5.5$ to $10 \mathrm{mIU} / \mathrm{L})$, and $0.4 \%$ highly elevated $(10 \mathrm{mIU} / \mathrm{L})$. In girls, the determination of TSH serum level was performed more frequently than in boys. Moreover, the frequency of TSH testing increased with age. In the second TSH evaluation, TSH levels within the reference range were documented in $40 \%, 73.6 \%$, and $78.9 \%$ of those whose basic serum TSH was highly elevated, elevated, and low, respectively, and in $97 \%$ of those with normal initial TSH. Predictive factors for a sustained highly elevated TSH were initial TSH $>7.5 \mathrm{mIU} / \mathrm{L}$ $(P=0.014)$ and female gender $(P=0.047)$. The conclusions were as follows: (1) initial normal or slightly elevated TSH levels are likely to remain normal or spontaneously normalize without treatment; (2) patients with initial levels higher than $7.5 \mathrm{mU} / \mathrm{L}$, particularly girls, are at a greater risk for persistent abnormal TSH concentration.

Also Wasniewska et al. [10] in their prospective crosssectional controlled trial estimated the natural course of idiopathic SH. The authors analyzed 92 young patients whose clinical status, thyroid function, and autoimmunity were checked after 6, 12, and 24 months. All of the patients were asymptomatic, with no changes in BMI and height in follow-up. The 2-year prospective study showed that 
$38(41.3 \%)$ patients out of the entire cohort aged $<15$ years normalized their TSH values during follow-up, whilst, in $11(12 \%)$, the thyroid function deteriorated as demonstrated by the increase in TSH values to $10-15 \mathrm{mU} / \mathrm{L}$. Overall, the beneficial evolution was more manifested in pubertal patients than in prepubertal ones; it was not significantly affected by sex, FT4 levels, or familial antecedents of thyroid disease.

3.7. Thyroid Status in Puberty. In their cross-sectional controlled long-term study, Cerbone et al. [9] found that, in nine (six females; 25\%) of the $36 \mathrm{SH}$ children, puberty started and proceeded normally despite the persistence of untreated $\mathrm{SH}$ (2.2-7.5 yrs). Moreover, the final accomplished height of four of the children (two females) was more than adequate for the target height according to Tanner et al. [32]. In two (5.5\%) $\mathrm{SH}$ children, a possible constitutional delay of growth and puberty was suspected due to stature below $\mathrm{TH}$, with normal growth velocity, normal IGF1 concentration, GH peak after stimulation test within its reference range, and a delay of 2 years in bone maturation.

Rapa et al. [19] concluded that, in 19 (21.6\%) out of 88 children with $\mathrm{SH}$, serum TSH concentrations were not influenced by puberty status as determined by visual inspection according to the Tanner scale.

3.8. SH and Vitiligo. Cho et al. [28] investigated the relationship between vitiligo and thyroid gland dysfunctions. The authors retrospectively compared the thyroid function of 254 children with vitiligo and 122 healthy controls. The results showed that there was no significant disparity in the range of thyroid disease between healthy children and the cohort with vitiligo.

3.9. SH and TSH-R Gene Mutations. Rapa et al. [19] in their multicenter study made an attempt to establish not only the clinical characteristics and biochemical parameters but also TSH-R gene variations in children and adolescents with $\mathrm{SH}$. A total of 19 known variations in TSH-R gene were found in the study population, of which eight were nonsynonymous mutations found in ten patients. Those patients had a 1.9fold higher prevalence of positive family history of thyroid diseases than patients without any detected mutation.

3.10. SH in In Vitro Fertilization Children. Two case-control studies $[29,30]$ assessed the condition of the thyroid gland in in vitro fertilization (IVF) babies. Onal et al. [29] analyzed the TSH concentration in newborns at the age of 2-4 weeks. They examined 98 babies conceived after classic IVF, of whom 10 had elevated serum TSH level above $6.5 \mathrm{mUI} / \mathrm{L}$ and were diagnosed with SH. Thyrotropin-releasing hormone (TRH) tests were executed in all subjects, both in the study group and in controls. The control group consisted of 10 naturally conceived babies with euthyroid hyperthyrotropinemia. The peak of TSH response was measured at $20 \mathrm{~min}$. TSH response to TRH was considered normal if the peak TSH level was between 5 and $25 \mathrm{mIU} / \mathrm{L}$. A peak value above $25 \mathrm{mIU} / \mathrm{L}$ was considered exaggerated, whilst that below $5 \mathrm{mIU} / \mathrm{L}$ was considered suppressed. The baseline TSH levels were
$8.08 \pm 1.66 \mathrm{mUI} / \mathrm{L}$ in the study group and $7.55 \pm 0.80 \mathrm{mUI} / \mathrm{L}$ in controls. At $20 \mathrm{~min}$ of the TRH test, the TSH serum concentration was significantly higher in the population of IVF newborns than in naturally conceived controls: $55.57 \pm$ $14.31 \mathrm{mUI} / \mathrm{L}$ versus $19.37 \pm 3.47 \mathrm{mUI} / \mathrm{L}$.

Sakka et al. [30] studied 106 IVF children aged 4 to 14 yrs and 68 randomly selected non-IVF controls of the same age. The cut-off TSH serum concentration of $5.0 \mathrm{mU} / \mathrm{L}$ was a criterion for the second TSH determination and thyroid ultrasound. Children were considered to have persistent hyperthyrotropinemia if the TSH level was confirmed. Persistent elevations of circulating TSH were observed in seven IVF children and in none of the controls. The higher $\mathrm{SH}$ incidence in in vitro fertilization babies than in controls did not depend on birth weight, gestational age, small for gestational age and adequate for gestational age status, breastfeeding duration, gender, current age, BMI-SDS or pubertal status of IVF children, BMI, thyroiditis, maternal polycystic ovary syndrome (PCOS), gestational diabetes mellitus, parity, arterial hypertension during pregnancy, or smoking of their mothers.

3.11. Reference Range for TSH. Kratzsch et al. [31] observed 1,004 children divided into 3 age groups in order to determine the reference ranges for thyroid function tests in children and adolescents and to identify factors that may influence the limits of these intervals. Puberty was followed by a rise of TSH, FT3, and T3 levels. T4 and t-uptake were significantly higher in girls than in boys. The exclusion of children with increased TPO-Ab and TG-Ab had no significant influence on the lower and upper limit of the reference interval for TSH. The authors concluded that age, BMI-SDS, white blood cells count, and gender should be taken into account when diagnosing SH based on serum TSH concentration.

\section{Discussion}

The definition of $\mathrm{SH}$ is purely biochemical [1]. The establishment of the upper limit of normal TSH in different age groups poses a challenge [33]. Furthermore, there is no consensus about the TSH level at which treatment should be considered. In most of the analyzed publications, serum TSH over $4.2 \mathrm{mUI} / \mathrm{L}$ was used as $\mathrm{SH}$ cut-off point [8-11, 13, 14, 16, 19-21, 23-30]. L-thyroxine therapy was not administered in any of the cited studies. Screening for thyroid disorders has become more common in recent years, thus leading to $\mathrm{SH}$ being diagnosed more frequently, especially in younger subjects.

The analysis of SH-related symptoms was based on two different types of studies. In the first type, $\mathrm{SH}$ confirmation was sought as a result of symptoms/clinical presentation [12$18,26-30]$. In the second type, $\mathrm{SH}$ was diagnosed incidentally and only then followed by clinical assessment for typical manifestation [9-11, 19-25]. The natural history of thyroid function was described in two studies $[8,10]$. The question arises whether $\mathrm{SH}$ actually causes symptoms or whether their presence is a coincidence. The answer may influence the decision regarding pharmacotherapy. Older $\mathrm{SH}$ patients can 
present cardiovascular risks, depressed systolic function, and left ventricular diastolic dysfunction and may report reduced exercise tolerance [34]. What about the clinical manifestation and consequences in younger populations?

We only analyzed articles in which subjects presented biochemically pure $\mathrm{SH}$. The prevalence of $\mathrm{SH}$ in children and adolescents in the analyzed literature was between $2.9 \%$ and $14.1 \%[8,21,25]$ and could be modified by weight. In the obese and overweight population, the percentage of $\mathrm{SH}$ was significantly higher, reaching even more than $30 \%[15,16]$, hyperthyrotropinemia being a consequence rather than the cause of a higher BMI. Studies analyzing height, growth velocity, and puberty did not confirm a negative influence of $\mathrm{SH}$ [9].

$\mathrm{SH}$ should be distinguished from physiological or transiently increased TSH in the serum, especially during the recovery phase from nonthyroidal illnesses or after subacute, painless thyroiditis. For this reason, serum TSH should be checked after 3-6 months [31]. This observation was confirmed by two studies examining the natural course of $\mathrm{SH}[8,10]$. One of them, a retrospective analysis of 121,052 children, indicated TSH above $7.5 \mathrm{mIU} / \mathrm{L}$ and female sex as predictive factors for fixed $\mathrm{SH}$ [8]. Spontaneous reversibility of elevated TSH was found in $41-73 \%$ of subjects $[8,10]$.

Rarely, higher serum TSH concentrations are seen in patients with TSH-receptor mutations causing mild TSH resistance. It can affect up to $0.6 \%$ of white population and should be suspected in positive family history of elevated TSH, not coexisting with thyroid autoimmunity [35].

\section{Conclusions}

Subclinical hypothyroidism is a common clinical problem. According to the definition, $\mathrm{SH}$ is asymptomatic and detectable only by screening. However, there are a lot of controversies concerning population screening for $\mathrm{SH}$ as the benefits of therapy are not proven. Single placebo-controlled studies in older populations confirmed that screening and treatment by L-thyroxine improve the quality of life in only $1 \%$ of individuals [36]. The symptoms of SH are nonspecific. In some cases, the rise in TSH concentration was found incidentally, and only then the symptoms were looked for; in other cases, nonspecific complaints lead to the assessment of TSH. In both, there is no certainty concerning the connection between higher TSH levels and the presence of symptoms.

Our study limitation is searching in PubMed base only and restricting the search to English language. Probably a systematic search across multiple databases would yield more results. However, as the quality of most studies is poor and no definite conclusions can be drawn, randomized, largescale studies in children and adolescents are warranted to determine the best care for patients with $\mathrm{SH}$.

\section{Conflict of Interests}

The authors declare that there is no conflict of interests regarding the publishing of this paper.

\section{Authors' Contribution}

All authors equally contributed to the paper.

\section{Acknowledgment}

The authors wish to thank Sandra K. Lindon for proofreading the paper.

\section{References}

[1] B. Biondi and D. S. Cooper, "The clinical significance of subclinical thyroid dysfunction," Endocrine Reviews, vol. 29, no. 1, pp. 76-131, 2008.

[2] T. Wu, J. W. Flowers, F. Tudiver, J. L. Wilson, and N. Punyasavatsut, "Subclinical thyroid disorders and cognitive performance among adolescents in the United States," BMC Pediatrics, vol. 6, article 12, 2006.

[3] A. Monzani, F. Prodam, A. Rapa et al., "Endocrine disorders in childhood and adolescence. Natural history of subclinical hypothyroidism in children and adolescents and potential effects of replacement therapy: a review," European Journal of Endocrinology, vol. 168, no. 1, pp. R1-R11, 2012.

[4] P. B. Kaplowitz, "Subclinical hypothyroidism in children: normal variation or sign of a failing thyroid gland?" International Journal of Pediatric Endocrinology, vol. 2010, Article ID 281453, 8 pages, 2010.

[5] M. J. O'Grady and D. Cody, "Subclinical hypothyroidism in childhood," Archives of Disease in Childhood, vol. 96, no. 3, pp. 280-284, 2011.

[6] B. Gencer, T.-H. Collet, V. Virgini et al., "Subclinical thyroid dysfunction and the risk of heart failure events: an individual participant data analysis from 6 prospective cohorts," Circulation, vol. 126, no. 9, pp. 1040-1049, 2012.

[7] D. S. Cooper and B. Biondi, "Subclinical thyroid disease," The Lancet, vol. 379, no. 9821, pp. 1142-1154, 2012.

[8] L. Lazar, R. B. D. Frumkin, E. Battat, Y. Lebenthal, M. Phillip, and J. Meyerovitch, "Natural history of thyroid function tests over 5 years in a large pediatric cohort," Journal of Clinical Endocrinology and Metabolism, vol. 94, no. 5, pp. 1678-1682, 2009.

[9] M. Cerbone, C. Bravaccio, D. Capalbo et al., "Linear growth and intellectual outcome in children with long-term idiopathic subclinical hypothyroidism," European Journal of Endocrinology, vol. 164, no. 4, pp. 591-597, 2011.

[10] M. Wasniewska, M. Salerno, A. Cassio et al., "Prospective evaluation of the natural course of idiopathic subclinical hypothyroidism in childhood and adolescence," European Journal of Endocrinology, vol. 160, no. 3, pp. 417-421, 2009.

[11] M. W. Kuiper and E. J. van der Gaag, "Subclinical hypothyroidism in children can normalize after changes in dietary intake," Food and Nutrition Sciences, vol. 3, no. 3, pp. 411-416, 2012.

[12] T. Reinehr, A. Isa, G. de Sousa, R. Dieffenbach, and W. Andler, "Thyroid hormones and their relation to weight status," Hormone Research, vol. 70, no. 1, pp. 51-57, 2008.

[13] V. N. Baş, Z. Aycan, S. Y. Ağladıoğlu, and H. N. P. Kendirci, "Prevalence of hyperthyrotropinemia in obese children before and after weight loss," Eating and Weight Disorders, vol. 18, no. 1, pp. 87-90, 2013. 
[14] A. Grandone, N. Santoro, F. Coppola, P. Calabrò, L. Perrone, and E. M. del Giudice, "Thyroid function derangement and childhood obesity: an Italian experience," BMC Endocrine Disorders, vol. 10, article 8, 2010.

[15] B. Wolters, N. Lass, and T. Reinehr, "TSH and free triiodothyronine concentrations are associated with weight loss in a lifestyle intervention and weight regain afterwards in obese children," European Journal of Endocrinology, vol. 168, no. 3, pp. 323-329, 2013.

[16] K. V. S. Hari Kumar, A. Verma, J. Muthukrishnan, and K. D. Modi, "Obesity and thyrotropinemia," Indian Journal of Pediatrics, vol. 76, no. 9, pp. 933-935, 2009.

[17] S. Shalitin, M. Yackobovitch-Gavan, and M. Phillip, "Prevalence of thyroid dysfunction in obese children and adolescents before and after weight reduction and its relation to other metabolic parameters," Hormone Research, vol. 71, no. 3, pp. 155-161, 2009.

[18] G. Radetti, W. Kleon, F. Buzi et al., "Thyroid function and structure are affected in childhood obesity," Journal of Clinical Endocrinology and Metabolism, vol. 93, no. 12, pp. 4749-4754, 2008.

[19] A. Rapa, A. Monzani, S. Moia et al., "Subclinical hypothyroidism in children and adolescents: a wide range of clinical, biochemical, and genetic factors involved," Journal of Clinical Endocrinology \& Metabolism, vol. 94, no. 7, pp. 2414-2420, 2009.

[20] A. T. Ergür, Y. Taner, E. Ata, E. Melek, E. E. Bakar, and T. Sancak, "Neurocognitive functions in children and adolescents with subclinical hypothyroidism," Journal of Clinical Research in Pediatric Endocrinology, vol. 4, no. 1, pp. 21-24, 2012.

[21] H. Chen, Q. Xi, H. Zhang et al., "Investigation of thyroid function and blood pressure in school-aged subjects without overt thyroid disease," Endocrine, vol. 41, no. 1, pp. 122-129, 2012.

[22] T. Ittermann, M. Thamm, H. Wallaschofski, R. Rettig, and H. Völzke, "Serum thyroid-stimulating hormone levels are associated with blood pressure in children and adolescents," Journal of Clinical Endocrinology \& Metabolism, vol. 97, no. 3, pp. 828-834, 2012.

[23] M. Cerbone, D. Capalbo, M. Wasniewska et al., "Cardiovascular risk factors in children with long-standing untreated idiopathic subclinical hypothyroidism," The Journal of Clinical Endocrinology \& Metabolism, vol. 99, no. 8, pp. 2697-2703, 2014.

[24] J. Zhang, R. Jiang, L. Li et al., "Serum thyrotropin is positively correlated with the metabolic syndrome components of obesity and dyslipidemia in Chinese adolescents," International Journal of Endocrinology, vol. 2014, Article ID 289503, 7 pages, 2014.

[25] R. di Mase, M. Cerbone, N. Improda et al., "Bone health in children with long-term idiopathic subclinical hypothyroidism," Italian Journal of Pediatrics, vol. 38, no. 1, article 56, 2012.

[26] R. Fallah, M. Mirouliaei, N. Bashardoost, and M. Partovee, "Frequency of subclinical hypothyroidism in 5- to 15-yearold children with migraine headache," Journal of Pediatric Endocrinology and Metabolism, vol. 25, no. 9-10, pp. 859-862, 2012.

[27] B. Ekici and A. N. Cebeci, "The debate on the link between subclinical hypothyroidism and childhood migraine: is initial endocrinological evaluation necessary for children with migraine?” Acta Neurologica Belgica, 2014.

[28] S. B. Cho, J. H. Kim, J. M. Park, Y. K. Park, and S. H. Oh, "Vitiligo in children and adolescents: association with thyroid dysfunction," Journal of the European Academy of Dermatology and Venereology, vol. 25, no. 1, pp. 64-67, 2011.
[29] H. Onal, O. Ercan, E. Adal, A. Ersen, and Z. Onal, "Subclinical hypothyroidism in in vitro fertilization babies," Acta Paediatrica, vol. 101, no. 6, pp. 248-252, 2012.

[30] S. D. Sakka, A. Malamitsi-Puchner, D. Loutradis, G. P. Chrousos, and C. Kanaka-Gantenbein, "Euthyroid hyperthyrotropinemia in children born after in vitro fertilization," Journal of Clinical Endocrinology and Metabolism, vol. 94, no. 4, pp. 13381341, 2009.

[31] J. Kratzsch, G. Schubert, F. Pulzer et al., "Reference intervals for TSH and thyroid hormones are mainly affected by age, body mass index and number of blood leucocytes, but hardly by gender and thyroid autoantibodies during the first decades of life," Clinical Biochemistry, vol. 41, no. 13, pp. 1091-1098, 2008.

[32] J. M. Tanner, R. H. Whitehouse, and M. Takaishi, "Standards from birth to maturity for height, weight, height velocity and weight velocity: British children, 1965," Archives of Disease in Childhood, vol. 41, pp. 613-635, 1966.

[33] K. M. Kahapola-Arachchige, N. Hadlow, R. Wardrop, E. M. Lim, and J. P. Walsh, "Age-specific TSH reference ranges have minimal impact on the diagnosis of thyroid dysfunction," Clinical Endocrinology, vol. 77, no. 5, pp. 773-779, 2012.

[34] B. Biondi, "Cardiovascular effects of mild hypothyroidism," Thyroid, vol. 17, no. 7, pp. 625-630, 2007.

[35] N. Jordan, N. Williams, J. W. Gregory, C. Evans, M. Owen, and M. Ludgate, "The W546X mutation of the thyrotropin receptor gene: potential major contributor to thyroid dysfunction in a Caucasian population," Journal of Clinical Endocrinology and Metabolism, vol. 88, no. 3, pp. 1002-1005, 2003.

[36] M. Abu-Helalah, M. R. Law, J. P. Bestwick, J. P. Monson, and N. J. Wald, "A randomized double-blind crossover trial to investigate the efficacy of screening for adult hypothyroidism," Journal of Medical Screening, vol. 17, no. 4, pp. 164-169, 2010. 


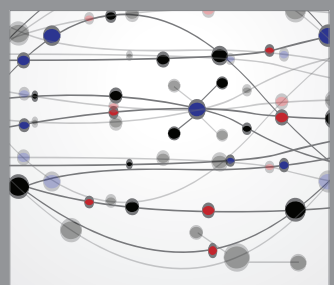

The Scientific World Journal
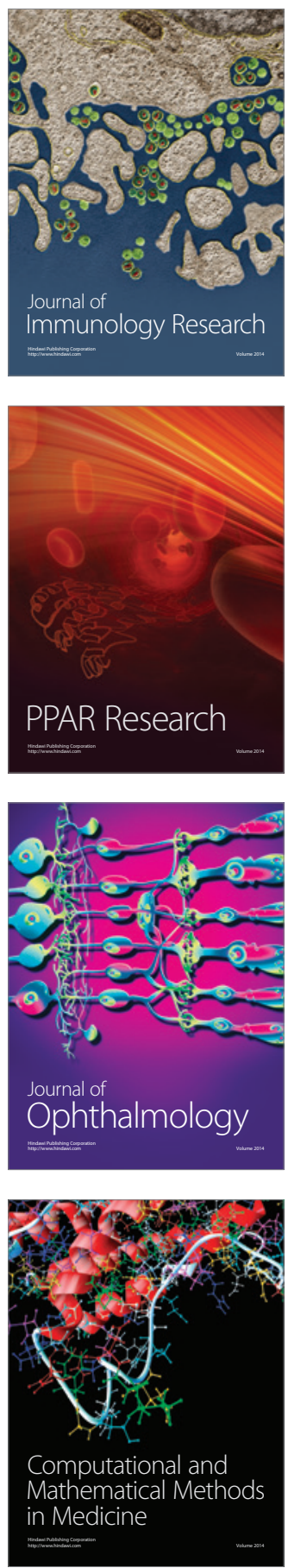

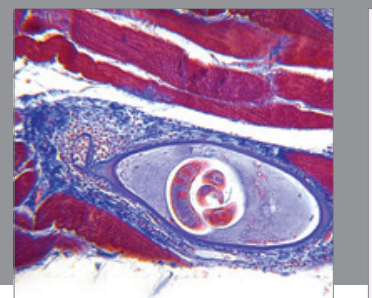

Gastroenterology

Research and Practice
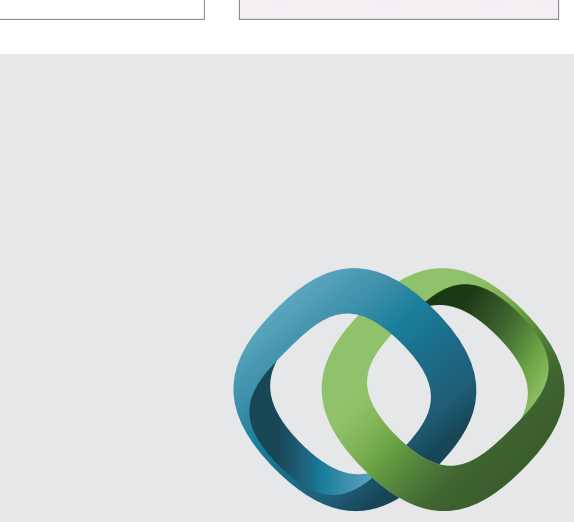

\section{Hindawi}

Submit your manuscripts at

http://www.hindawi.com
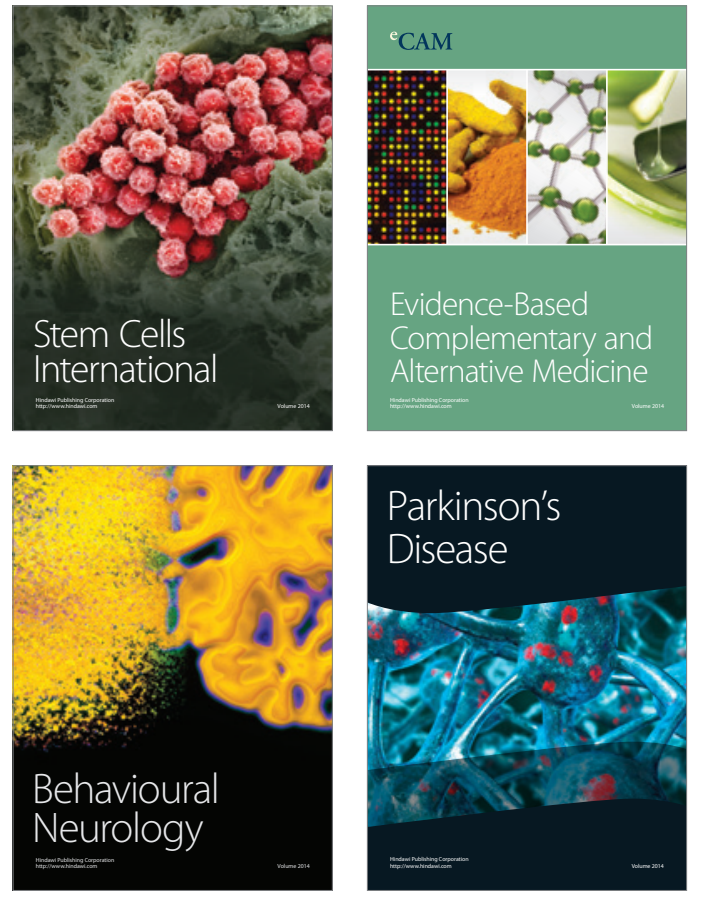
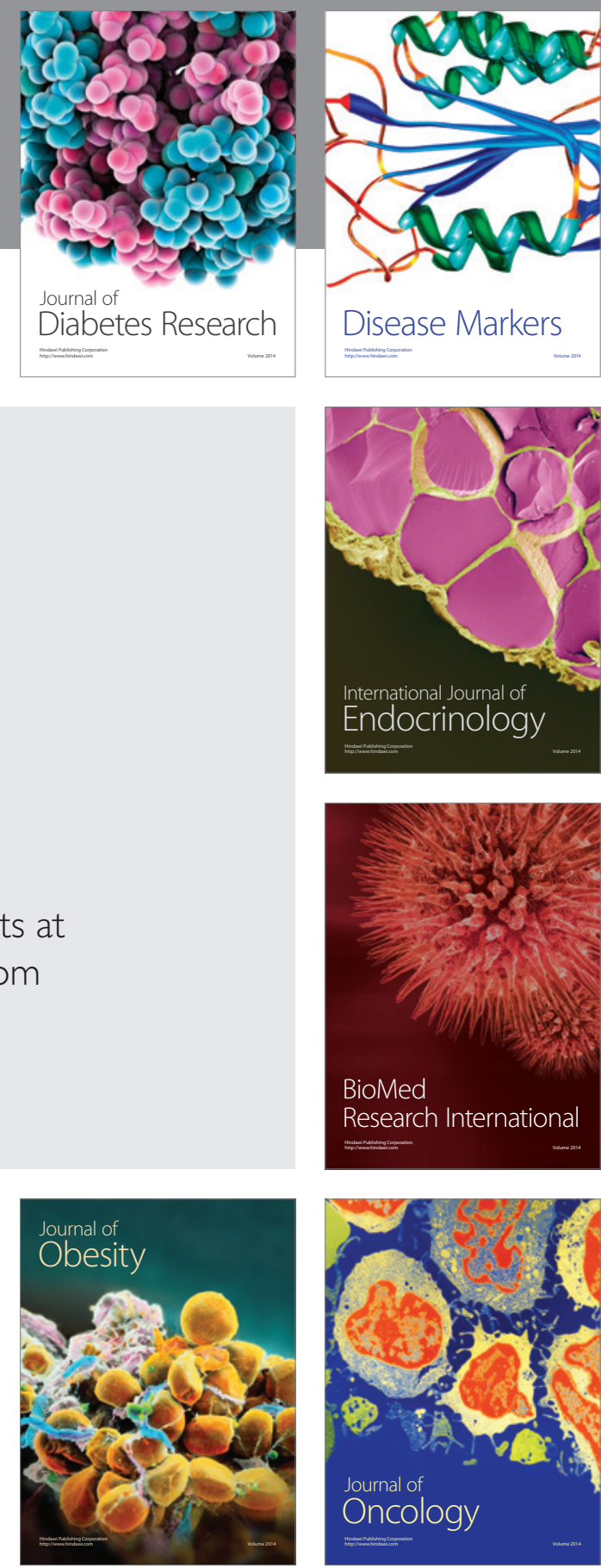

Disease Markers
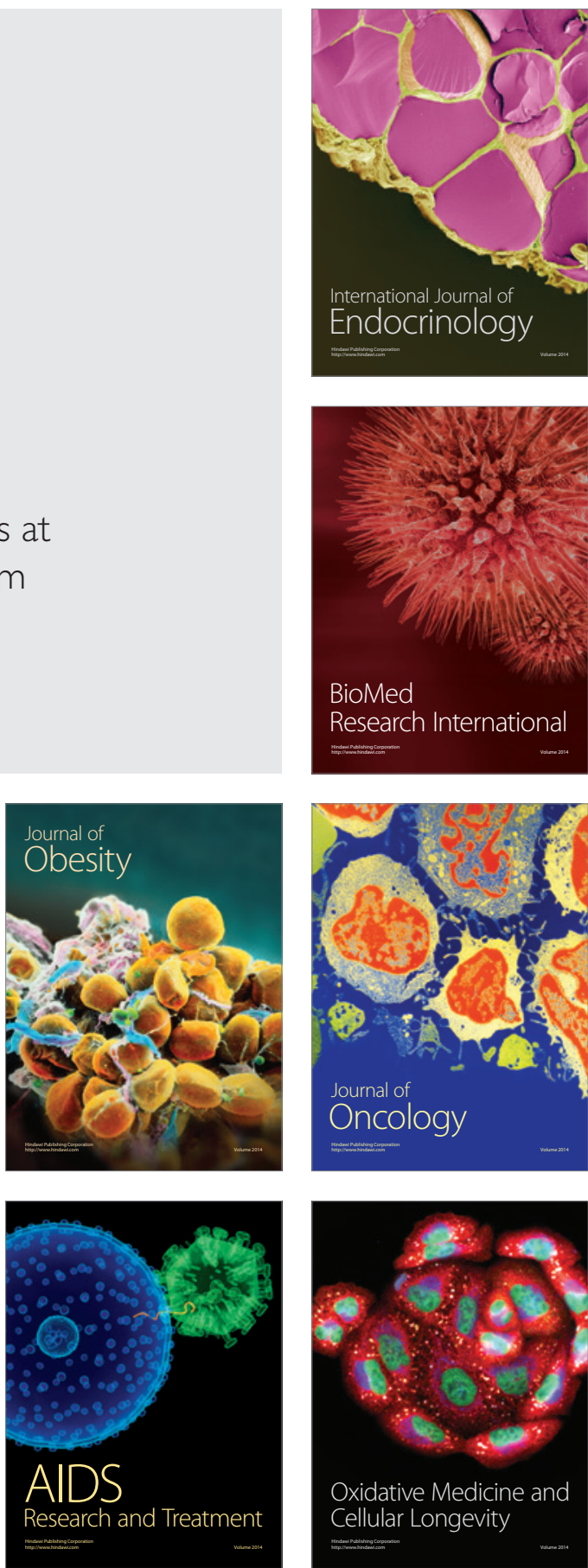